\title{
Oligopeptide Production from Alanine Monomer by Pulsed Corona Discharge Plasma in Ambient and Supercritical Argon
}

\author{
Koichi Nagafuchi ${ }^{1}$, Atsushi Nagira ${ }^{1}$, Hidenori Akiyama ${ }^{1}$, Mitsuru Sasaki ${ }^{1, *}$, Kunio Kawamura ${ }^{2}$ \\ ${ }^{1}$ Graduate School of Science and Technology, Kumamoto University, Kumamoto, Japan \\ ${ }^{2}$ Hiroshima Shudo University, Hiroshima, Japan \\ *Corresponding author: msasaki@kumamoto-u.ac.jp
}

Received December 21, 2012; Revised June 18, 2013; Accepted June 19, 2013

\begin{abstract}
Supercritical carbon dioxide and electric discharge plasma could be one of reaction or extraction media for green chemical processes. Supercritical fluid is an attractive solvent due to its unique characteristic and transport physical properties. Taking advantage of these characteristics, this has been extensively investigated for extraction and chemical conversion technology. Although density of a supercritical fluid is high like a liquid, it is mobile like a gas because of low viscosity. On the other hand, the electron energy generated by electric discharge plasma in high pressure and a high-density domain generally serves as an index depending on the field intensity and medium density which were supplied to electric discharge space. From the above-mentioned viewpoint, the synergistic effect of combining electric pulsed plasma and supercritical fluids is expected as a new reaction process which may be able to enhance chemical reaction without using any catalysts. In this research, production of protein-like substance by pulse electric discharge plasma was carried out using amino acid in supercritical carbon dioxide. Moreover, nano pulse electric discharge plasma was also formed in supercritical fluids; reaction of amino acid was also observed. Aiming at establishment of a new reaction field, we did fusion research of supercritical fluids and electric discharge plasma. In this experiment, argon at the pressurized state was used as a reaction medium. Initially, alanine aqueous solution of $0.1 \mathrm{~mol} / \mathrm{L}$ was loaded to a plasma reactor (inner volume: $600 \mathrm{~mL}$ ) and reached under the desired conditions (290-308 K and 1-10.8 MPa). They were then analyzed by high-performance liquid chromatography (HPLC) and matrix-assisted laser desorption time of flight mass spectroscopy (MALDI TOF-MS). The possibility of polymerization of alanine was realized from the compound after electric pulsed discharge plasma processing.
\end{abstract}

Keywords: pulsed corona discharge plasma, non-thermal equilibrium plasma, oligopeptide, alanine, supercritical argon

\section{Introduction}

In general, it is very difficult to generate electric discharge plasma in high-pressure and high-density fluids. It strongly depends on the medium density in the electric discharge space. The electron energy used as an index is proportional to the supplied reciprocal product of the field intensity and medium density. The electric discharge space serves as an important element when an average free path is short and when generating sufficient high electrolysis and high energy electrons increases the collision ionization coefficient. Non-thermal equilibrium plasma by the nanosecond pulse electric discharge is able to offer the activated species by electric discharge, without disturbing atmospheric fluid. The consideration of hydrothermal origin theory discussed the evolution a life from an inorganic substance. This hypothesis is supported by various observed fact. The water temperature spouting from the hydrothermal vent into the deep sea water is at around $400^{\circ} \mathrm{C}$, suggesting that the deep sea water near the hydrothermal vent is in supercritical state. Protein is one of the most important and common substances present in living things, and is considered to be responsible for the origin of life. Periodic simulation of the vent system involving hot water and amino acids to and from the sea surface resulted into formation of oligopeptides, suggesting the possibility of appearance of life in the primitive earth. However, the yields of the oligopeptides from amino acid alanine were typically $0.1-1 \%$ by these simulations [1,2,3,4,5].

The viewpoint of the demand to an environmentally harmless organic integrated process to an organic solvent cannot be found. Efficient preparation of oligopeptides is attractive. Novel electrochemical polymerization may be attained by electric discharge plasma without any catalyst. Some reactions like the conversion of glyceride were induced by using electric discharge plasma generated with supercritical carbon dioxide [6]. In addition, polymerization of phenol was performed using pulse electric discharge plasma in hydrothermal state without using any catalyst [7]. Carbon dioxide is the most utilized solvent in supercritical fluid technologies. It acts like an ordinary organic solvent such as hexane, ethanol, and methanol by simply adjusting the temperature and 
pressure. In addition, it is an environmentally friendly and generally regarded as safe solvent. For this reason, supercritical carbon dioxide $\left(\mathrm{SCCO}_{2}\right)$ can be used for extraction of natural products, chemical conversions without organic solvents and additives. $\mathrm{SCCO}_{2}$ is the state above its critical point $\left(\mathrm{Tc}=304 \mathrm{~K}, \mathrm{Pc}_{\mathrm{c}}=7.38 \mathrm{MPa}\right)$ and behaves like a dense gas. On the other hand, electric discharge plasma has a high energy electron, ion, and the various reaction species that are radical. Those can enhance chemical reaction without catalysts. Therefore, electric discharge plasma in combination with supercritical fluids without using any catalyst will become an environmentally attractive reaction field. The electric discharge feature of supercritical carbon dioxide was investigated by using high-voltage direct-current electric discharge $[8,9]$. Pulsed power technology has been widely used worldwide, in order to stabilize high-pressure conditions like that of a supercritical fluid and to make electric discharge plasma. Pulsed power technology can instantly inject huge electric power into the fluid. From its advantages, the generation of electrical discharge plasma in supercritical fluids may achieve an enhanced reaction process due to rich radicals produced by electrical discharge plasma. For the above reasons, the concern with SCFs with electrical discharge plasma has been growing in recent years.

Recently, Kawamura et al. have investigated the elongation reaction of oligoalanine under hydrothermal conditions [10]. According to this research, oligopeptides of alanine including large amounts of by-product "diketopiperazine", which is generally formed by thermolysis of alanine dimer or trimer, have been produced at $275^{\circ} \mathrm{C}$ and $15 \mathrm{MPa}$. The reaction for hydrothermal supplies in protein is from amino acid. It is one of the most important materials after the appearance of the life in relation to polypeptide. In recent years, according to literature regarding reaction behavior of amino acids under hydrothermal conditions by Kawamura et al., it was found that peptide bonds were able to generate from alanine tetramer plus monomer to form alanine hexamer. However, it is important issue that the thermal instability of amino-peptide has been relating to the efficiency of peptide reaction process. Although it is very interesting, this was a result of a reaction invlving catalysts to promote chemical reaction. Moreover, under the present circumstances, the conversion of amino acids is in low level and the reaction mechanism for oligopeptide production from amino acids has not been clarified. Especially, there are no reports about the oligopeptide production from alanine dimer or monomer as starting materials.

For improving these problems, we proposed the pulsed corona discharge treatment in argon (Ar) at the ambient and supercritical state. We at first employed the needle (negative electrode) to plane (positive electrode) electrodes for generating non-uniform electric field and the electrode gap was set to $1 \mathrm{~mm}$. They were made of tungsten and stainless steel, respectively. Moreover, we are using the small ceramics of a coefficient of linear expansion for the support supporting the high-voltage of an electrode, and installation side. Possibility of polymerization was suggested by making nano pulse discharge plasma form in supercritical fluid. We will generate high energy field by using thermal equilibrium plasma. Furthermore, we can suppose pinpoint over with needle to plate electrode. Consequently, corona discharge using non-thermal equilibrium plasma is found to be one of suitable reaction tools for this reaction.

Here we investigated the application of pulsed corona discharge plasma under ambient or pressurized Ar using a coaxial cylindrical electrode in order to understand reaction behavior of alanine monomer. The products obtained were analyzed by HPLC and MALDI TOF-MS with the experimental findings indicating the possibility to generate oligopeptides from alanine monomer by the assist reaction of pulsed corona discharge under noncatalytic supercritical Ar atmosphere.

\section{Experimental and Analytical Sections}

\subsection{Materials}

DL- $\alpha$-alanine (99.0\%) obtained from Wako Pure Chemicals Industries Ltd. (Osaka, Japan) were used as starting materials. Ala-Ala (99.0\%) obtained from SIGMA ALDRICH Japan Science Industries Ltd (Tokyo, Japan).

$\mathrm{NaH}_{2} \mathrm{PO}_{4}, \mathrm{CH}_{3}\left(\mathrm{CH}_{2}\right)_{5} \mathrm{SO}_{3} \mathrm{Na}$ and methanol used as a determination for HPLC analysis obtained from Wako Pure Chemicals Industries Ltd. (Osaka, Japan). Carbon dioxide (high purity) which was supplied from Uchimura Sanso Co., Ltd. (Kumamoto, Japan) was used as a solvent. Other chemicals were also purchased from this company and they were used without further purification.

\subsection{Experimental Procedures}

Figure 1 shows the schematic diagram of the experimental apparatus which consists of electric pulse discharge in a supercritical fluids. The stainless steel SUS316 test reactor (AKICO Co., Japan) with the total inner volume of $900 \mathrm{~mL}$, and its pressure capacity and temperature are up to $30 \mathrm{MPa}$ and $573 \mathrm{~K}$, respectively. The high voltage lead feed-through was a specially-designed bushing of PEEK resin and was sealed with double Orings. There is a refrigeration unit in reactor both ends. The PEEK resin used this time is an aromatic series system plastic and has the outstanding characteristics, such as heat resistance, fire retardancy, radioactivity-proof, and insulation. The plasma reactor was improved by the both viewpoints of the reaction volume and electrode configuration as follows: 1) to make internal volume of the reactor smaller by using PEEK resin having excellent properties in heat resistance and electrical insulation properties; 2) to change the needle-to-plate configuration into the coaxial electrode system in order to achieve the generation of non-thermal equilibrium plasma and its control in dense fluids. Possibility of the occurrence of any chemical reactions was expected by increasing the electric discharge surface area.

In this experiment, Ar gas at the pressurized state was used as a reaction medium. Initially, alanine aqueous solution of $0.1 \mathrm{~mol} / \mathrm{L}$ was loaded to a plasma reactor (inner volume: $600 \mathrm{~mL}$ ). After reached the desired temperature (290, 293, 298 or $307 \mathrm{~K})$ and pressure $(1,5$ or $10.8 \mathrm{MPa})$, a pulsed high voltage was applied to the electrode in the test reactor to produce electric discharge plasma at the constant temperature and pressure. After a given processing time has passed, the reaction solution was 
collected from a wire electrode part and then the remaining fraction inside the reactor was washed-out with distilled water. The products collected were analyzed by HPLC and MALDI-TOF-MS.

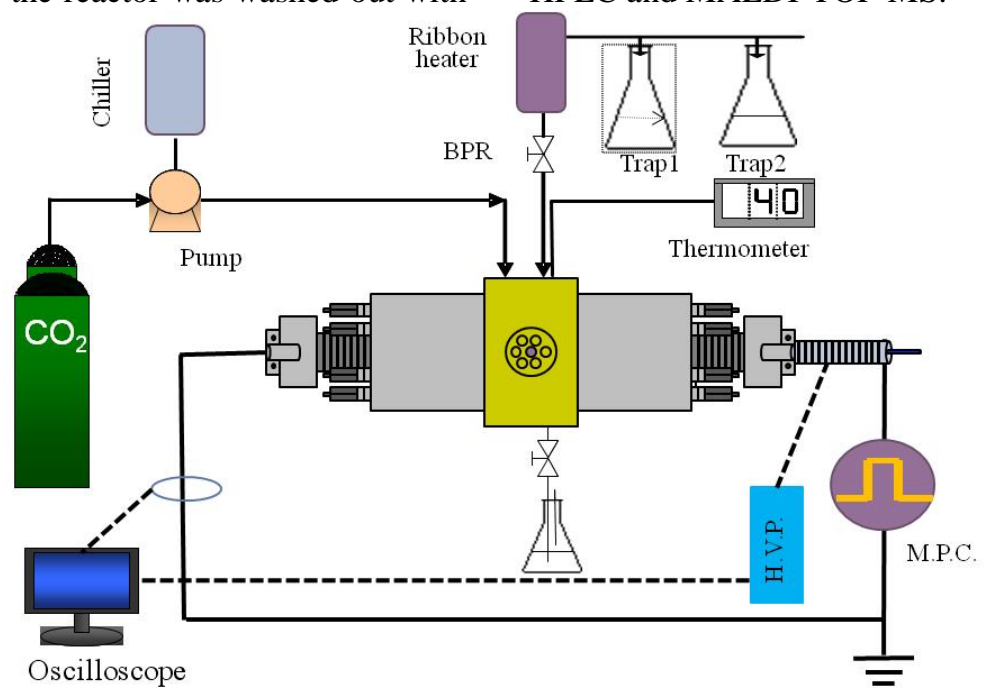

Figure 1. Schematic diagram of the experimental apparatus used in this study

\subsection{Analytical Methods}

The quantitative analysis of reaction solutions was carried out by an HPLC system with a multi-wavelength Photodiode Array Detector (SPD-M10A VP) in the following conditions.

Column: Inertsil ODS-3,

Flow rate: $0.7 \mathrm{~mL} / \mathrm{min}$,

Eluent: A) $5 \mathrm{mM} \mathrm{NaH} \mathrm{PO}_{4}, 3.6 \mathrm{mM} \mathrm{CH}_{3}\left(\mathrm{CH}_{2}\right)_{5} \mathrm{SO}_{3} \mathrm{Na}$ in water (pH2.65); B) $10 \mathrm{mM} \mathrm{NaH} \mathrm{PO}_{4}, \quad 7.2 \mathrm{mM}$ $\mathrm{CH}_{3}\left(\mathrm{CH}_{2}\right)_{5} \mathrm{SO}_{3} \mathrm{Na}$ in $50 \%$ Methanol (pH2.7),

Temperature: $308 \mathrm{~K}$

Mode: Binary gradient A/B (time) $=70 / 30$ (0 min) 40/60 (15 min ) - 40/60 (25 min $)$ - 70/30 (26 min) - 70/30 (40 $\mathrm{min})(\mathrm{v} / \mathrm{v})$,

Detector: Diode Array Detector (SPD-M10A VP),

Wavelength: 220nm,

Injection volume: $10 \mathrm{uL}$

The qualitative analysis of the product was also conducted by a MALDI-TOF-MS system. Analytical conditions were as follows: Acceleration (V) $+25 \mathrm{kV}$, nitrogen laser 337nm 3 ns pulse width, laser probe: Laser Probe Rm-3700 Universal Radiometer, matrix: 2,5Dihydroxybenzoic acid (DHB).

\section{Results and Discussion}

In order to explore optimum conditions where the corona discharge was generated in Ar atmosphere, pulsed discharge experiments were carried out at various temperatures, pressures, pulse frequency and treatment times. The discharge behavior was observed through an observation sapphire window (o.d. $=15 \mathrm{~mm}$ ) of the batchtype view cell using a camera with recording speed of 30 films/s. As a result, it was found that corona discharge was generated in supercritical Ar at $5 \mathrm{MPa}, 290 \mathrm{~K}$ and 160 pps for a suitable treatment time as shown in Figure 2 (left-hand side photo). For extended treatment time or more severe operating conditions, the corona discharge hardly occurred and shifted to bright arc discharge as shown in Figure 2 (right).
Chemical reaction experiments for alanine aqueous solution of $0.1 \mathrm{~mol} / \mathrm{L}$ were conducted at $5 \mathrm{MPa}$ and $290 \mathrm{~K}$. The HPLC chromatograms obtained from aqueous solutions before and after the discharge treatment with nano-pulsed discharges were compared as shown in Figure 3.

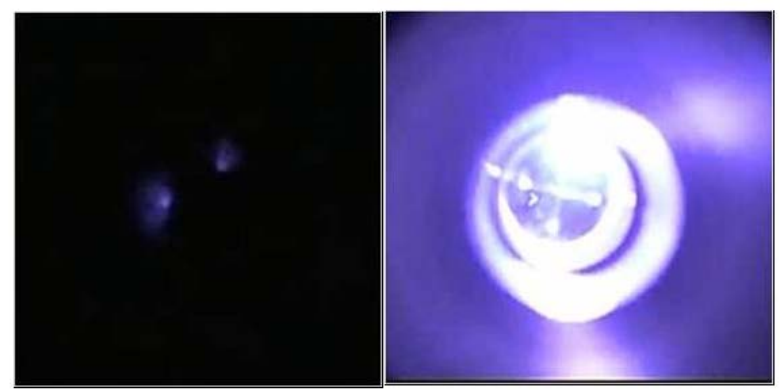

Figure 2. Observations of nano-plused discharge plasma in supercritical $\mathrm{Ar}$ at $5.0 \mathrm{MPa}, 290 \mathrm{~K}$ and $160 \mathrm{pps}$ for a short treatment time (left) and at 10.8MPa, 308K, 160 pps (right)

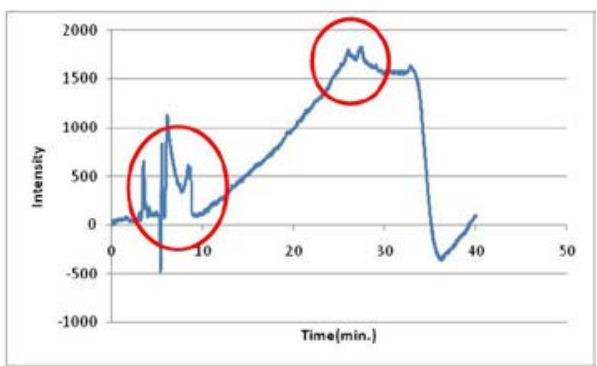

Figure 3. HPLC chlomatogram of the product solution obtained after the pulsed corona discharge treatment in $\mathrm{Ar}$ at $298 \mathrm{~K}, 5 \mathrm{MPa}$ and 10,000 shots

The peak (15 minutes) before discharge processing was lost. Moreover, the fall (5 min.) of relative luminescence intensity was accepted by HPLC analysis. The small luminescence peak was checked from $26 \mathrm{~min}$. to $28 \mathrm{~min}$. A possibility that the oligopeptide reaction is induced is realized. We have not resulted by identification of compound. From these analysis results, the possibility of oligomerization of the amino acids by the assistant 
reaction of the pulse electric discharge without any catalyst was suggested.

Results of the MALDI-TOF-MS and HPLC analyses of the reaction products obtained by the pulsed corona discharge treatment in ambient argon atmosphere are shown in Figure 4 and Figure 5, respectively. The peak of alanine was detected from the analysis result of MALDITOF-MS to processing before, and after. Electric discharge processing has doubled Intensity. The possibility of promotion of the reaction of alanine was suggested. Possibility that the dimer and trimer of alanine were made was suggested.

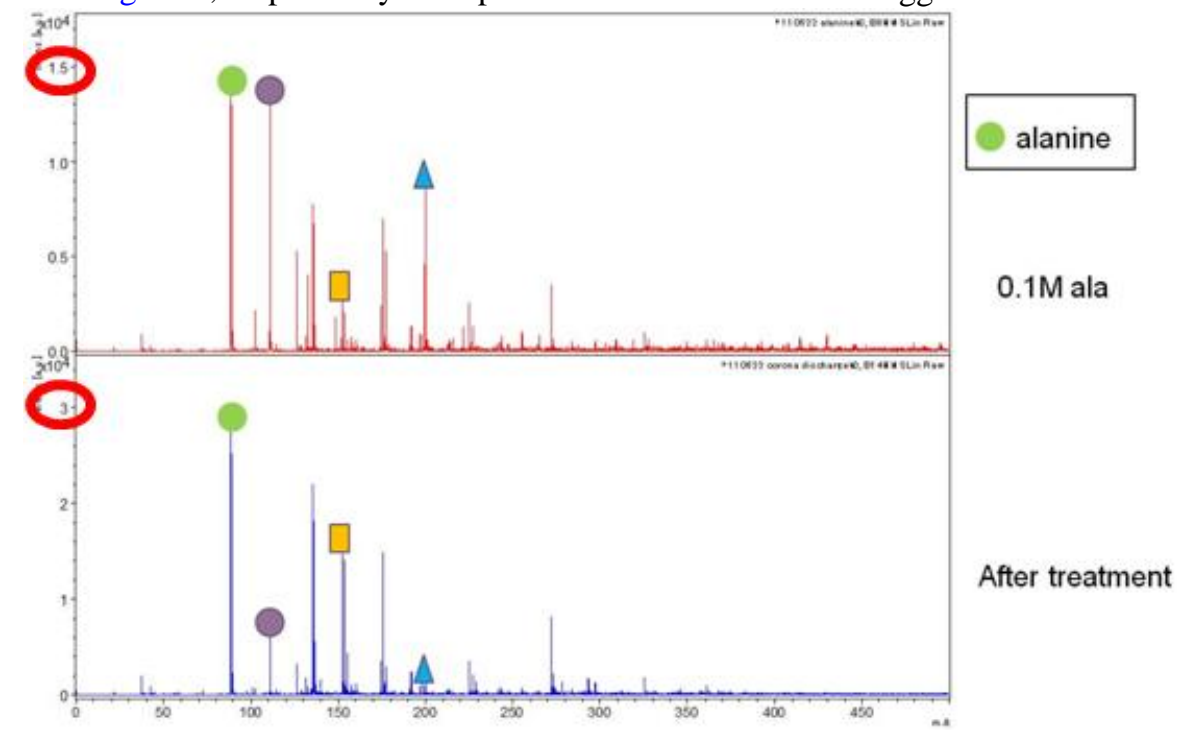

Figure 4. A MALDI-TOF-MS spectra of the product obtained by the treatment in Ar at 293K, 1MPa and 25,000 shots

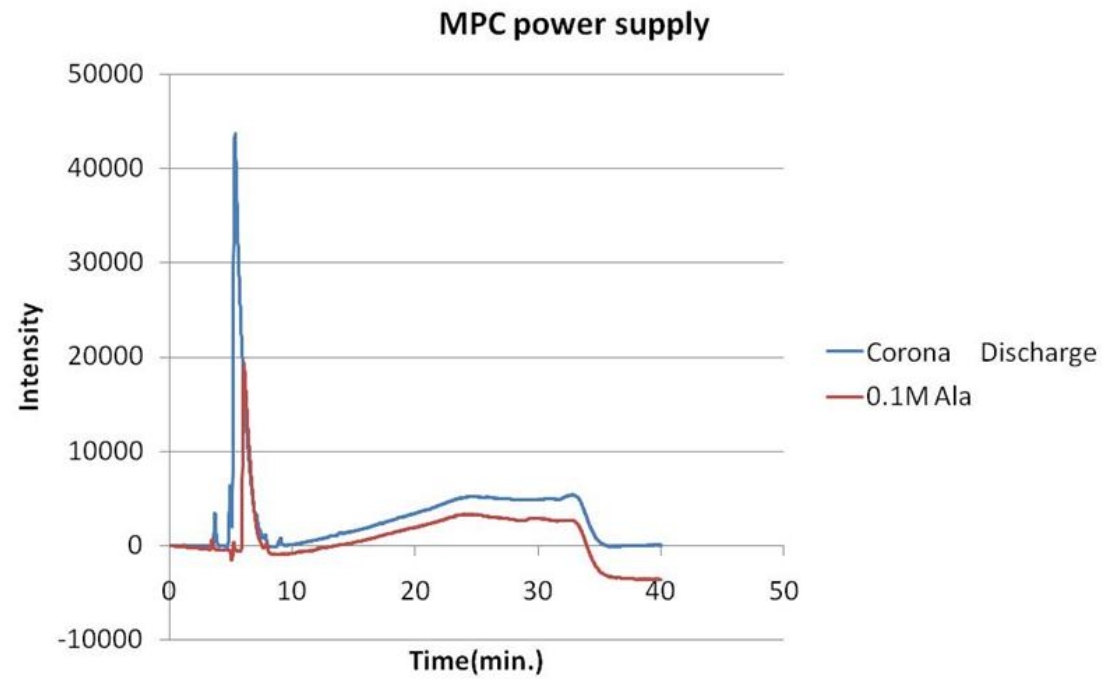

Figure 5. HPLC chromatograms of untreated alanine aqueous solution and the product solution obtained after the pulsed corona discharge treatment in Ar at 293K, 1.0MPa and 25,000 shots

The peak of alanine in the HPLC chromatogram (see Figure 5) was detected from the analysis result of HPLC in 6 minutes (before processing). The big peak was detected in 7 minutes (after electric discharge processing). The small peak was detected in 4 and 9 minutes. Suggested that alanine polymerized and decomposition.

\section{Conclusions}

Reaction behavior of alanine in ambient and supercritical $\mathrm{Ar}$ was investigated using a batch-type pulsed electric (corona) discharge plasma reactor. As a result, we found that oligopeptides were formed from alanine monomer, which shows the possibility of oligomerization of the amino acid by pulse electric discharge assisted reaction without catalyst. The peak intensity after pulse electric discharge processing was the result of differing from HPLC and data original at both MALDI - TOF - MS. Future research plans will focus on the use of nanosecond pulse electric discharge plasma in supercritical state for the reaction experiment of alanine in a coaxial reactor.

\section{Acknowledgments}

This work was supported by the Kumamoto University Global COE Program "Global Initiative Center for Pulsed Power Engineering". It was also supported by the Scientific Research on Innovative Areas " Frontier science of interactions between plasmas and nano-interfaces" supported by the Ministry of Education, Culture, Sports, Science and Technology, Japan. 


\section{References}

[1] Corliss, J. B.; Baross, J. A.; Hoffman, S. E., Oceanol. "An hypothesis concering the relationship between submarine hot springs and th origin of life on Earth”, Acta (supplement) 1981, 4, 59-69.

[2] Baross, J. A.; Hoffman, S. E.” Submarine hydrothermal vents and associated gradient environments as sites for the origin and evolution of life”, Origins Life 1985, 15, 327-354.

[3] Nisbet, E. G. "Origin of life: RNA and hot-water springs", Nature 1986, 322, 206.

[4] Yanagawa, H.; Egami, F., Proc. Jpn. Acad., Ser. B 1984, 54, 331336.

[5] Imai, E.; Honda, H.; Hatori, K.; Matsuno, K., Origins Life Evol. Biosphere, 1999, 29, 249-259.

[6] Goto, M.; Sasaki, M.; Kiyan, T.; Fang, T.; Roy, B. C.; Namihira, T.; Akiyama, H.; Hara, M., J Physics: Conference Series, 121, 2008.
[7] Kiyan, T.; Sasaki, M.; Ihara, T.; Namihira,T.; Hara, M.; Goto, M.; Akiyama, H., Plasma, Processes and Polymers, Vol. 6, No. 11, 2009, pp. 778-785

[8] Kiyan, T.; Uemura, A.; Roy, B. C.; Namihira, T.; Hara, M.; Sasaki, M.; Goto, M.; Akiyama, H., IEEE Trans. on Plasma Science, 35, 2007, 656-662.

[9] Kiyan, T.; Takade, M.; Namihira, T.; Hara, M.; Sasaki, M.; Goto, M.; Akiyama, H., IEEE Trans. on Plasma Science, 36, 2008, 821827.

[10] Kawamura, K.; Nishi, T.; Sakiyama, T.; J. Am. Chem. Sci., 127, 2005, 522-523

[11] Wahyudion; Kanetake, T.; Sasaki, M.; Goto, M., Chem. Eng. Technol. 30, 2007, No. 8, 1113-1122.

[12] Hobo, T.; Oguma K.: Rikoukeiikibunsekinokiso (The basics of instrumental analysis of science and technology) (Asakurashoten,Tokyo,2001) pp. 31-32 [in Japanese]. 